\title{
Innovation and Entrepreneurship in a Space-time Framework Based on the Case of Poland
}

\begin{abstract}
Although innovation and entrepreneurship have been subjects of researchers' interests for years, nevertheless both the concepts and the relationships between them remain controversial. Since different indices are accepted for the measurement of these categories, the question is whether the relationship between them depends on the choice of measures. The aim of the paper is to indicate the innovation impact on the entrepreneurial process with regard to different measures of these concepts. The research was conducted with the use of regression analyses as a method and yearly data for Polish regions in the period 2003-2018. At the initial stage of the research as many measures of innovation and entrepreneurship as possible were introduced into the estimation of regression function parameters. Statistically insignificant variables were deleted at later stages of research. This research confirms that innovativeness and entrepreneurship are broad concepts and the relationship between them depends on the choice of measures. Saturation with enterprises and saturation with start-ups seem to be more sensitive to innovation than other measures of entrepreneurship. Innovation seen as input, measured by spending on research and development $(\mathrm{R} \& \mathrm{D})$ has a bigger influence on entrepreneurship than the output innovation measured by revenues from innovative activities. Considering a relatively low level of explanatory power of innovations, it seems they are not the only factor influencing entrepreneurship.
\end{abstract}

Keywords: entrepreneurship; innovation; R\&D activities; regions of Poland

Received: 13 June 2019

Accepted: 29 September 2019

\section{Suggested citation:}

Gawel, A. (2019). Innovation and entrepreneurship in a space-time framework based on the case of Poland. Przedsiębiorczość - Edukacja [Entrepreneurship - Education], 15(2), 24-39. doi: $10.24917 / 20833296.152 .2$

\section{Introduction}

Innovation and entrepreneurship have been the subject of interest for researchers and policymakers for many years, but these phenomena are still variously defined and measured, and this leads to a discussion about the relationship between them. This article 
aims to look at how the adopted measures of innovation and entrepreneurship determine the results of the relationship between them. At the initial stage as many measures of innovation and entrepreneurship were adopted as possible, to eliminate those statistically insignificant at the later stages of research, and ultimately get a complete picture of the phenomenon. The selected spatial and temporal perspective referred to Poland, taking into account the annual data of the Central Statistical Office for Polish voivodeships (administrative units) in the period 2003-2018. Then, using a regression method, those independent variables reflecting innovative activity which turned out to be irrelevant in explaining entrepreneurship as a dependent variable, were removed. The resulting image of relations confirms that innovation is a broad concept, and the adoption of different definitions of innovation and entrepreneurship can lead to varying results in the relationships between them. Saturation with enterprises and saturation with start-ups tend to be more sensitive to innovation than other measures of entrepreneurship. At the same time, innovations in terms of input, measured by spending on research and development (R7D), affect entrepreneurship more strongly than innovations in the revenue system, related to innovative activity, that is the scale of enterprises implementing innovation. At the same time, judging by the relatively low level of volatility in explaining the formation of entrepreneurship, innovation is not the only factor affecting it.

\section{Innovations and the main area of research}

The pioneer of research on innovation is Joseph Schumpeter (Polish edition, 1960), who defined them as new combinations in the economy, being new products, production technologies, new markets, market organisations or sources of supply of raw materials. At the same time, according to Schumpeter's theoretical approach (1960), innovations are a source of profit, a cause of business cycles and economic growth. In the broad concept of innovation, it is assumed that for companies, these are new or improved solutions to existing ideas or products. In the narrow sense, innovation means new products or solutions on the market (Koellinger, 2008; Lejpras, 2014). Innovations are also treated as an interactive process that relates to the behaviour of enterprises, as well as planning and implementing changes (Nauwelaers, Wintjes, 2002).

The innovativeness of enterprises is associated with their involvement in the development of new markets, mainly export ones (North, Smallbone, 2000), or a stage of company development, with start-ups considered more innovative (Huergo, Jaumandreu, 2004). Innovation also seems to be affected by its inertia and economy of scale. Innovative enterprises that already have patents are more likely to apply for more licenses than those that do not have them (Lejpras, 2014). The innovativeness of enterprises is influenced by factors such as concentration in industry, intensity of export and import activities, and the level of economic development of a country (Bhattacharya, Bloch, 2004; Koellinger, 2008).

Research on innovativeness often explores the relationship between R\&D expenditure and innovation, although the results of these studies are not precise. The intensity of $\mathrm{R} \& \mathrm{D}$ activities is measured both by the degree of implementation and expenditure in the company's overall turnover (Lejpras, 2014). According to some studies, spending on R\&D is a factor that positively affects innovation (Acs, Audretsch, 1988; Bhattacharya, Bloch, 2004). However, the results of other studies indicate a lack of a connection with product or market innovations, and its negative impact on the innovation process 
(Simonen, McCann, 2008). Research and development activities allow the development of assets specific only to a given company, thanks to which competitors cannot imitate these resources and enable the company to gain a competitive advantage (Esteve-Pérez, Mañez-Castillejo, 2008).

The links between innovation and entrepreneurship result from the fact that the introduction of new products or processes can take place in two ways, either through implementations made by start-ups or by developing the scope of activity of companies stabilised on the market (Auerswald, 2008). Both solutions have their pros and cons, but it seems essential that the innovation activity of existing companies is in competition with the innovation activity of new enterprises. The innovativeness of existing companies is a factor which may lead to difficulties in imitating them, and as a consequence lead to fewer start-ups being developed (Luttmer, 2007).

Innovation is indicated as one of the main theoretical currents in defining entrepreneurship, along with equating it with a tendency to take risks and using business opportunities. Explaining the essence of entrepreneurship through the prism of innovation takes place in several contexts, such as the implementation of new solutions in the entrepreneurial process related to the establishment of new business entities, as a strategic dimension in the entrepreneurial orientation of enterprises, or as a feature of enterprises and entrepreneurs (Gawel, 2013, 2019 et seq.).

\section{Entrepreneurship - creation and development of start-ups}

In the narrow sense adopted in the article, the essence of entrepreneurship is identified with the creation and development of start-ups, because all dimensions of entrepreneurial competencies are involved in this process. Research indicates, however, that many factors of a different nature affect the rates of enterprise creation, survival and collapse, including the innovativeness of both the enterprise itself and the innovation of its environment. It is worth noting, however, that the literature on the subject indicates different results of research on the impact of factors on the creation and survival of a company (Yang, Bossink, Peverelli, 2017).

Creating and developing a new company is an interaction between the enterprise and its environment. As a result, the factors shaping the entrepreneurial process can be divided into internal ones, related to the entrepreneur and the structure of the created entity, and into external factors associated with the economic, social or cultural environment. Internal factors describing an entrepreneur include such features as the need for achievement, internal control placement, initiative, proactivity and the need for self-fulfillment (Korunka, Frank, Lueger, 2003), attitude towards risk (Caliendo, Fosse, Kritikos, 2009), level of education (Naudé et al., 2008) or previous entrepreneurial experience (Capelleras, Greene, 2008). For the survival of a newly created company, in addition to the personal characteristics of the entrepreneur, internal resources are also significant. The literature indicates the level of employee qualifications, internal financial capital and R\&D (Yang, Bossink, Peverelli, 2017).

The second group of factors affecting the rate of creation of start-ups is related to its environment. They include market profit rates, the economic size of the region, its economic characteristics and growth rate, the use of external support, population density, the rate of creation of start-ups in the area, imperfections in financial markets related to 
liquidity barriers and moral hazard, and the type of industrial regime (Audretsch, Fritsch, 2002; Capelleras, Greene, 2008; Naudé et al. 2008; Paulson, Townsend, Karaivanou, 2006; Wagner, Sternberg, 2004). Access to human capital in the region is also essential, including the level of employee qualifications or the level of their education (Armington, Acs, 2002).

The subject of entrepreneurship research is also the issue of the failure or survival of a start-up on the market. The risk of failure changes over time. It increases after starting a business and reaches its maximum after a year or two, and then decreases (Strotmann, 2007). The probability of failure is influenced by factors related to the entrepreneur's personality, company structure and environment (Box, 2008). The first includes their education and previous professional experience (Mengistae, 2006), as well as the entrepreneur's private financial assets that eliminate the problem of liquidity barriers (Schäfer, Talavera, 2009) and assets acquired through research and development (Esteve-Pérez, Mañez-Castillejo, 2008). On the other hand, factors affecting the risk of the failure related to the environment include operating in industries with higher minimum efficiency, characterised by lower demand (Strotmann, 2007), and the activity in sectors with higher competitive pressure (Mengistae, 2006).

Innovation is one of the factors that influence the process of creating and developing a start-up, both at the individual and environmental levels. Innovation is treated as one of the entrepreneur's personality traits while traits such as university education, previous $\mathrm{R} \& \mathrm{D}$ experience and strong motivation to implement new ideas significantly increases the likelihood that a new company will undertake innovative activity, especially in R\&D (Arvanitis, Stucki, 2012; Honjo, Kato, Okamuro, 2014). This impact seems to be positive and long-term, while the impact of the financial capital of the newly formed company on $\mathrm{R} \& \mathrm{D}$ expenditure is mainly of a short-term nature (Wang, 2014).

The innovativeness of new enterprises in a given region seems to depend on the regional environment in such aspects as the availability of essential resources, including venture capital funds and qualified staff, or a regional knowledge base, including the innovative activity of companies operating in the region or universities and research institutes in the area. However, such an environment does not necessarily lead to a higher likelihood of survival of a start-up (Fritsch, Schroeter, 2011).

The concept of technological regimes explains the diverse impact of the environment on innovativeness. It assumes the existence of two types of regime: entrepreneurial and routine. In the entrepreneurial regime, a relatively large number of new enterprises emerges that introduce innovations to the market. This regime shows creative destruction, under which new innovative enterprises enter the market and displace stable companies. In such a regime, the market is more open to innovations introduced by start-ups. In the routine regime, on the other hand, the rate of creation of new companies is relatively low, with a creative accumulation, i.e. a relatively stable number of enterprises. In this case, innovations are introduced to the market to a greater extent by stabilised enterprises (Audretsch, Fritsch, 2002; Lin, Huang, 2008; Peneder, 2008).

A specific situation related to the location of R\&D activities is the impact of the location of universities and research institutes as entities involved in R\&D. Research results indicate that the level of $\mathrm{R} \& \mathrm{D}$ activity, and the geographical proximity of universities and research institutes increase the likelihood of start-ups entering the market, especially in technology industries, although they do not seem to affect the survival of companies (de Silva, McComb, 2012). 
However, a more in-depth look into the problem of a region's innovation impact indicates that it may not be entirely clear-cut. Innovations in the region, measured by patent activity in metropolitan areas, negatively affect the survival of newly established companies starting their business with 1-3 employees. This impact decreases as the size of the company increases, including for start-ups with four employees it is irrelevant, and with a larger number of employees, the region's innovativeness increases the chances of survival (Tsvetkova, Thill, Strumsky, 2014).

Research assumptions of the relationship between innovation and entrepreneurship

The multi-faceted concepts of innovation and entrepreneurship result in the adoption of various measures of operationalisation. This raises questions to what extent measures of innovation and entrepreneurship affect the results of research on the relationships between them; and how the impact of innovation on entrepreneurship is shaped depending on the measures adopted. The presented research results are an attempt to answer the above questions. Hence, the broadest possible number of available indicators operationalising both phenomena was considered. Using such regression function parameters as a research method, initially measures of both entrepreneurship as a dependent variable and innovation as an independent variable were successively inserted into the general form of the function. Then, independent variables that were statistically insignificant were removed.

With the broadest possible understanding of the concept of entrepreneurship and the largest number of its measures, the following five have been accepted:

- rate of establishing new enterprises (ST) - the share of the number of start-ups in the total number of enterprises,

- enterprise liquidation rate $(\mathrm{SU})$ - the share of the number of liquidated enterprises in the total number of enterprises,

- net enterprise rate $(\mathrm{SN})$ - the difference between the rate of creation of start-ups and the rate of liquidation of enterprises,

- enterprise saturation (NPRZ) - number of enterprises per 1000 inhabitants,

- saturation with newly created enterprises (NSU) - the number of start-ups per 1000 inhabitants.

The relationships between the above measures can be represented by Figure 1 .

Even greater complexity is found when defining and measuring innovation. Initially, the largest available number of innovation measures were adopted. They can be divided into output and input measures, which either perceive innovation through the prism of obtained results or expenditures. The resulting approach is related to two aspects: the sale of innovative products and the number of innovative enterprises, additionally adjusted by size. The input approach indicates expenditures on $\mathrm{R} \& \mathrm{D}$ and innovation in relation to other input. Details on innovation measures adopted at the initial stage of the study are summarised in Table 1.

After accepting the broadest possible spectrum of measures of entrepreneurship and innovation, the following research procedure was adopted. To linearise nonlinear relationships, all initial data were converted to natural logarithms. In the first step, the parameters of the regression function between successive measures of entrepreneurship as dependent variables and measures of innovation from each of the groups as independent variables were estimated, which gave a total of 20 primary functions (see Table 2). 
Figure 1. Relationships between measures of entrepreneurship

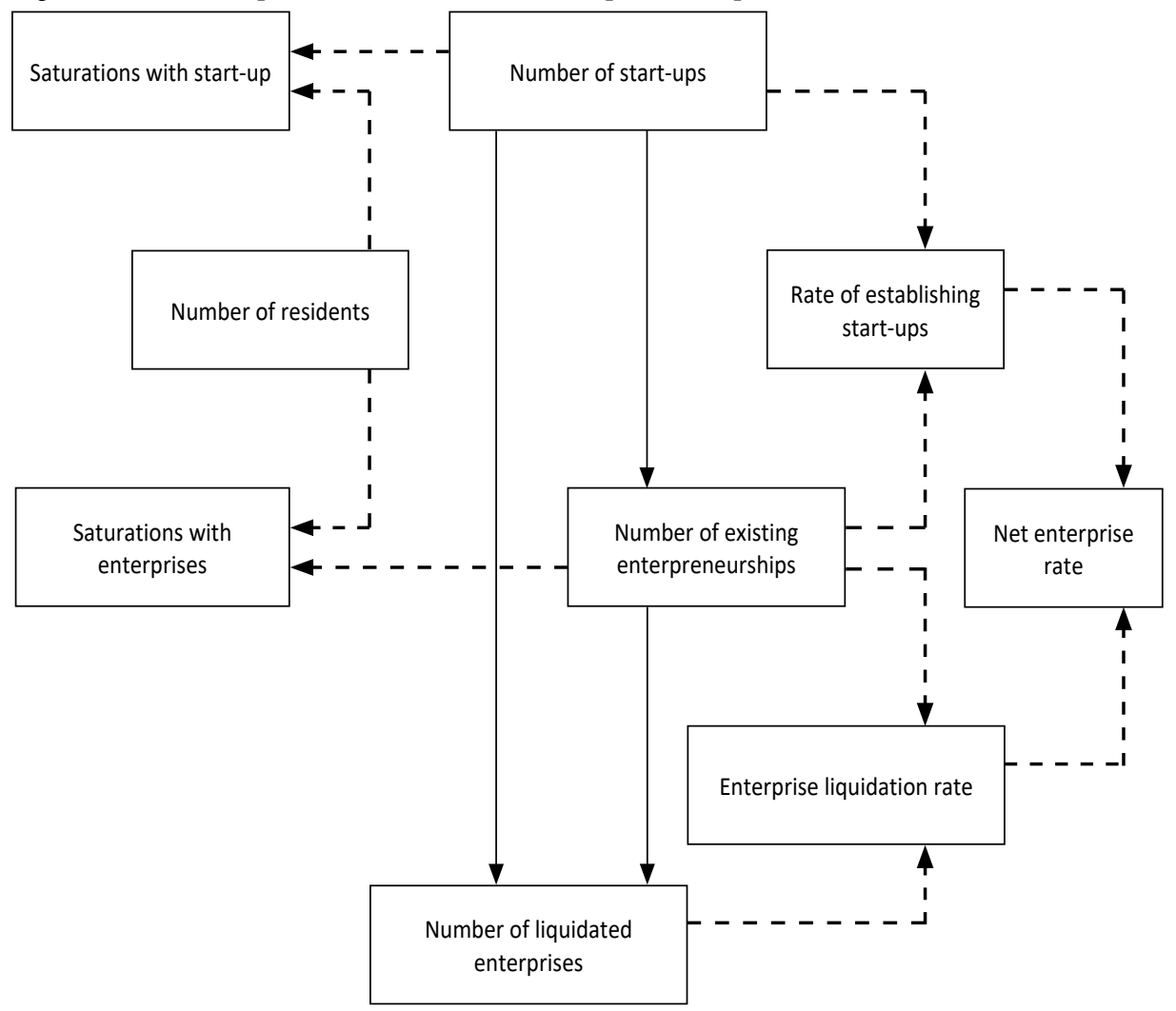

Source: own development

Then, the independent variables that were statistically insignificant based on the value of the significance coefficient $\mathrm{p}$ were successively removed from each function. The significance coefficient $\mathrm{p}$ determines the probability that results obtained from the dependence estimation is a coincidence: the lower its value, the more correct the estimated dependencies. The study adopted a threshold at a significance level of 0.1 , which means accepting a $10 \%$ probability of randomness of the results obtained. A p-value above 0.1 is interpreted as an indication that the function parameter is not statistically significant. The initially estimated function parameters are presented in Table 2.

After removing independent variables following the above assumptions, 19 forms of regression functions were obtained in which the parameters were statistically significant (see Table 3). Then, the R2 coefficient values were compared to identify the strongest relations. The regression functions which explained less than $10 \%$ of the variability of the dependent variable, i.e. towards which the R2 coefficient was lower than (0.10), were eliminated. Ultimately, this allowed 11 regression functions to be accepted as a basis for inferring a relationship between innovation and entrepreneurship. 
Table 1. Innovation measures adopted in the study

\begin{tabular}{|c|c|c|c|}
\hline \multicolumn{3}{|c|}{ Output measures of innovation } & \multirow[b]{2}{*}{$\begin{array}{l}\text { Input measures } \\
\text { of innovation }\end{array}$} \\
\hline $\begin{array}{l}\text { Net revenues from } \\
\text { sales of innovations }\end{array}$ & $\begin{array}{c}\text { Net revenues from } \\
\text { the sale } \\
\text { of innovations } \\
\text { by size class }\end{array}$ & $\begin{array}{l}\text { Share of innovative } \\
\text { enterprises in total } \\
\text { enterprises }\end{array}$ & \\
\hline $\begin{array}{l}\text { Net sales from innova- } \\
\text { tions in total revenues } \\
\text { (USPI) }\end{array}$ & $\begin{array}{l}\text { USPI in small, medi- } \\
\text { um and large enter- } \\
\text { prises (USPIM, USPIS, } \\
\text { USPID) } \\
\end{array}$ & $\begin{array}{l}\text { Share of innovative } \\
\text { enterprises in the ser- } \\
\text { vices sector (PIU) }\end{array}$ & $\begin{array}{l}\text { Share of expenditures } \\
\text { on R\&D in GDP } \\
\text { (NBR) }\end{array}$ \\
\hline $\begin{array}{l}\text { Net sales from market } \\
\text { innovations in total } \\
\text { revenues (USPIR) }\end{array}$ & $\begin{array}{l}\text { USPIR in small, } \\
\text { medium and large } \\
\text { enterprises (USPIRM, } \\
\text { USPIRS, USPIRD) }\end{array}$ & $\begin{array}{l}\text { Share of service en- } \\
\text { terprises introducing } \\
\text { new or significantly } \\
\text { improved products } \\
\text { (PIUNP) }\end{array}$ & $\begin{array}{l}\text { Expenditure on R\&D } \\
\text { per } 1 \text { professionally } \\
\text { active (NPRAC) }\end{array}$ \\
\hline $\begin{array}{l}\text { Net sales from innova- } \\
\text { tion for the enterprise } \\
\text { in total revenues } \\
\text { (USPIP) }\end{array}$ & $\begin{array}{l}\text { USPIP in small, } \\
\text { medium and large } \\
\text { enterprises (USPIPM, } \\
\text { USPIPS, USPIPD) }\end{array}$ & $\begin{array}{l}\text { Share of service en- } \\
\text { terprises introducing } \\
\text { new or significantly } \\
\text { improved products for } \\
\text { the market (PIUNPR) }\end{array}$ & $\begin{array}{l}\text { Expenditure on inno- } \\
\text { vation activities } \\
\text { in domestic expendi- } \\
\text { ture (NWN) }\end{array}$ \\
\hline $\begin{array}{l}\text { Net sales from inno- } \\
\text { vations for export in } \\
\text { total revenues (USPIE) }\end{array}$ & $\begin{array}{l}\text { USPIE in small, } \\
\text { medium and large } \\
\text { enterprises (USPIEM, } \\
\text { USPIES, USPIED) }\end{array}$ & $\begin{array}{l}\text { Share of service en- } \\
\text { terprises introducing } \\
\text { new or significantly } \\
\text { improved processes } \\
\text { (PIUNPROC) } \\
\end{array}$ & $\begin{array}{l}\text { Expenditures on } \\
\text { innovative activities } \\
\text { relative to gross ex- } \\
\text { penditure on fixed } \\
\text { assets (NŚT) } \\
\end{array}$ \\
\hline $\begin{array}{l}\text { Net sales from market } \\
\text { innovations for ex- } \\
\text { ports in total revenues } \\
\text { (USPIER) }\end{array}$ & $\begin{array}{l}\text { USPIER in small, } \\
\text { medium and large en- } \\
\text { terprises (USPIERM, } \\
\text { USPIERS, USPIERD) }\end{array}$ & $\begin{array}{l}\text { Share of innovative } \\
\text { enterprises in industry } \\
\text { (PIP) }\end{array}$ & \\
\hline \multirow[t]{4}{*}{$\begin{array}{l}\text { Net sales of products } \\
\text { from high and medi- } \\
\text { um-high technologies } \\
\text { in total revenues } \\
\text { (USPT) }\end{array}$} & & $\begin{array}{l}\text { Share of industrial en- } \\
\text { terprises introducing } \\
\text { new or significantly } \\
\text { improved products } \\
\text { (PIPNP) }\end{array}$ & \\
\hline & & $\begin{array}{l}\text { Share of industrial en- } \\
\text { terprises introducing } \\
\text { new or significantly } \\
\text { improved products for } \\
\text { the market (PIPNPR) }\end{array}$ & \\
\hline & & $\begin{array}{l}\text { Share of industrial en- } \\
\text { terprises introducing } \\
\text { new or significantly } \\
\text { improved processes } \\
\text { (PIPNPROC) } \\
\end{array}$ & \\
\hline & & $\begin{array}{l}\text { Share of innovative } \\
\text { enterprises in the total } \\
\text { number of enterprises } \\
\text { (UI) }\end{array}$ & \\
\hline
\end{tabular}

Source: own development 
Research results

The study was based on the situation in Poland, based on secondary data published by the Central Statistical Office. A spatial-temporal system was adopted, i.e. the dynamics in 2003-2018 and distribution in individual voivodeships were taken into account. It means that 256 observations of the measure of innovation and entrepreneurship were made. In the examined system, the average rate of creation of new enterprises was $8.48 \%$; the average failure rate $7.10 \%$; the average net rate $1.37 \%$; the average saturation with enterprises was 97.52 enterprises per 1000 inhabitants; and saturation with start-ups - 8.25 new companies per 1000 inhabitants.

Table 2. Parameters of the initial regression functions

\begin{tabular}{|c|c|c|c|c|c|}
\hline $\begin{array}{c}\text { Independent } \\
\text { variables }\end{array}$ & ST & SU & SN & NPRZ & NSU \\
\hline \multicolumn{6}{|c|}{ Net revenues from sales of innovations } \\
\hline No & 1 & 2 & 3 & 4 & 5 \\
\hline Constans & $\begin{array}{c}2.12477 \\
(\mathrm{p}=0.000)\end{array}$ & $\begin{array}{c}2.02192 \\
(\mathrm{p}=0.000)\end{array}$ & $\begin{array}{c}-0.187630 \\
(\mathrm{p}=0.8299)\end{array}$ & $\begin{array}{c}4.13533 \\
(\mathrm{p}=0.000)\end{array}$ & $\begin{array}{c}1.65492 \\
(p=0.000)\end{array}$ \\
\hline USPI & $\begin{array}{c}0.212332 \\
(\mathrm{p}=0.0047)\end{array}$ & $\begin{array}{c}0.234188 \\
(\mathrm{p}=0.1002)\end{array}$ & $\begin{array}{c}0.644822 \\
(\mathrm{p}=0.4481)\end{array}$ & $\begin{array}{c}0.330090 \\
(\mathrm{p}=0.0049)\end{array}$ & $\begin{array}{c}0.542421 \\
(\mathrm{p}=0.000)\end{array}$ \\
\hline USPIR & $\begin{array}{c}-0.135041 \\
(\mathrm{p}=0.0120) \\
\end{array}$ & $\begin{array}{c}-0.162790 \\
(\mathrm{p}=0.1107)\end{array}$ & $\begin{array}{c}-0.0610682 \\
(\mathrm{p}=0.9156)\end{array}$ & $\begin{array}{c}-0.291923 \\
(\mathrm{p}=0.0006)\end{array}$ & $\begin{array}{c}-0.426964 \\
(\mathrm{p}=0.000)\end{array}$ \\
\hline USPIP & $\begin{array}{c}-0.0836463 \\
(p=0.0215) \\
\end{array}$ & $\begin{array}{c}-0.0871872 \\
(p=0.2072)\end{array}$ & $\begin{array}{c}-0.241448 \\
(\mathrm{p}=0.5775)\end{array}$ & $\begin{array}{c}-0.150197 \\
(\mathrm{p}=0.0083)\end{array}$ & $\begin{array}{c}-0.233843 \\
(\mathrm{p}=0.0004)\end{array}$ \\
\hline USPIE & $\begin{array}{c}-0.0294112 \\
(\mathrm{p}=0.3225)\end{array}$ & $\begin{array}{c}-0.0372204 \\
(\mathrm{p}=0.5117)\end{array}$ & $\begin{array}{c}-0.234656 \\
(\mathrm{p}=0.4855)\end{array}$ & $\begin{array}{c}0.00636874 \\
(\mathrm{p}=0.8907)\end{array}$ & $\begin{array}{c}-0.0230425 \\
(\mathrm{p}=0.6644)\end{array}$ \\
\hline USPIER & \begin{tabular}{|c|}
0.0302744 \\
$(\mathrm{p}=0.253)$ \\
\end{tabular} & $\begin{array}{c}0.00458941 \\
(\mathrm{p}=0.9276)\end{array}$ & $\begin{array}{c}0.0247519 \\
(\mathrm{p}=0.9251)\end{array}$ & $\begin{array}{c}0.106990 \\
(\mathrm{p}=0.0102)\end{array}$ & $\begin{array}{c}0.137264 \\
(\mathrm{p}=0.0041)\end{array}$ \\
\hline USPT & $\begin{array}{c}-0.0260181 \\
(\mathrm{p}=0.1194) \\
\end{array}$ & $\begin{array}{c}-0.0440984 \\
(p=0.1666) \\
\end{array}$ & $\begin{array}{c}-0.0468226 \\
(\mathrm{p}=0.7880)\end{array}$ & $\begin{array}{c}0.0862811 \\
(\mathrm{p}=0.0011)\end{array}$ & $\begin{array}{c}0.0602629 \\
(\mathrm{p}=0.0442)\end{array}$ \\
\hline \multicolumn{6}{|c|}{ Net revenues from sales of innovations by size classes } \\
\hline No & 6 & 7 & 8 & 9 & 10 \\
\hline Constans & $\begin{array}{c}1.99350 \\
(\mathrm{p}=0.000) \\
\end{array}$ & $\begin{array}{c}2.00555 \\
(\mathrm{p}=0,000) \\
\end{array}$ & $\begin{array}{c}0.867182 \\
(\mathrm{p}=0.5404)\end{array}$ & $\begin{array}{c}4.82271 \\
(\mathrm{p}=0.000) \\
\end{array}$ & $\begin{array}{c}2.2110 \\
(\mathrm{p}=0.000)\end{array}$ \\
\hline USPIM & $\begin{array}{c}-0.0101319 \\
(\mathrm{p}=0.7816) \\
\end{array}$ & $\begin{array}{c}-0.0418179 \\
(p=0.5745)\end{array}$ & $\begin{array}{c}-0.628387 \\
(p=0.1161)\end{array}$ & $\begin{array}{c}-0.0764035 \\
(\mathrm{p}=0.2058)\end{array}$ & $\begin{array}{c}-0.0865354 \\
(\mathrm{p}=0.2041) \\
\end{array}$ \\
\hline USPIS & $\begin{array}{c}0.136733 \\
(p=0.3801)\end{array}$ & $\begin{array}{c}-0.154391 \\
(\mathrm{p}=0.6262)\end{array}$ & $\begin{array}{c}-0.999573 \\
(\mathrm{p}=0.5475)\end{array}$ & $\begin{array}{c}-0.0101838 \\
(\mathrm{p}=0.9683)\end{array}$ & $\begin{array}{c}0.126549 \\
(\mathrm{p}=0.6618)\end{array}$ \\
\hline USPID & $\begin{array}{c}0.117202 \\
(\mathrm{p}=0.0271) \\
\end{array}$ & $\begin{array}{c}0.166456 \\
(p=0.1217)\end{array}$ & $\begin{array}{c}0.582087 \\
(\mathrm{p}=0.3758)\end{array}$ & $\begin{array}{c}-0.0241332 \\
(\mathrm{p}=0.7806)\end{array}$ & $\begin{array}{c}0.0930684 \\
(\mathrm{p}=0.3418)\end{array}$ \\
\hline USPIRM & $\begin{array}{c}0.00246800 \\
(\mathrm{p}=0.9023) \\
\end{array}$ & $\begin{array}{c}-0.0247240 \\
(\mathrm{p}=0.5462) \\
\end{array}$ & $\begin{array}{c}0.590726 \\
(\mathrm{p}=0.0069)\end{array}$ & $\begin{array}{c}0.0200671 \\
(\mathrm{p}=0.5448)\end{array}$ & $\begin{array}{c}0.0225351 \\
(\mathrm{p}=0.5468) \\
\end{array}$ \\
\hline USPIRS & \begin{tabular}{|c|}
-0.0581138 \\
$(\mathrm{p}=0.4505)$ \\
\end{tabular} & $\begin{array}{c}0.0611415 \\
(\mathrm{p}=0.6964)\end{array}$ & $\begin{array}{c}0.376631 \\
(\mathrm{p}=0.6490)\end{array}$ & $\begin{array}{c}-0.0191089 \\
(\mathrm{p}=0.8802)\end{array}$ & $\begin{array}{c}-0.0772227 \\
(\mathrm{p}=0.5894)\end{array}$ \\
\hline USPIRD & $\begin{array}{c}-0.0599340 \\
(\mathrm{p}=0.1397)\end{array}$ & $\begin{array}{c}-0.0523225 \\
(p=0.5254)\end{array}$ & $\begin{array}{c}-0.171835 \\
(\mathrm{p}=0.7200)\end{array}$ & $\begin{array}{c}-0.0228980 \\
(p=0.7310)\end{array}$ & $\begin{array}{c}-0.0828320 \\
(\mathrm{p}=0.2714)\end{array}$ \\
\hline
\end{tabular}




\begin{tabular}{|c|c|c|c|c|c|}
\hline USPIPM & $\begin{array}{l}0.000999650 \\
(\mathrm{p}=0.9599)\end{array}$ & $\begin{array}{c}0.00715947 \\
(\mathrm{p}=0.8597)\end{array}$ & $\begin{array}{c}0.126785 \\
(\mathrm{p}=0.5706)\end{array}$ & $\begin{array}{c}0.00316047 \\
(\mathrm{p}=0.9232)\end{array}$ & $\begin{array}{c}0.00416012 \\
(\mathrm{p}=0.9104)\end{array}$ \\
\hline USPIPS & $\begin{array}{c}-0.0592290 \\
(\mathrm{p}=0.4881)\end{array}$ & $\begin{array}{c}0.0711877 \\
(\mathrm{p}=0.6822)\end{array}$ & $\begin{array}{c}1.02417 \\
(\mathrm{p}=0.2685)\end{array}$ & $\begin{array}{c}-0.156379 \\
(\mathrm{p}=0.2671)\end{array}$ & $\begin{array}{c}-0.215608 \\
(\mathrm{p}=0.1756)\end{array}$ \\
\hline USPIPD & $\begin{array}{c}-0.0514278 \\
(\mathrm{p}=0.0414)\end{array}$ & $\begin{array}{c}-0.0463767 \\
(\mathrm{p}=0.3635)\end{array}$ & $\begin{array}{c}-0.261419 \\
(\mathrm{p}=0.4247)\end{array}$ & $\begin{array}{c}0.0400406 \\
(\mathrm{p}=0.3323)\end{array}$ & $\begin{array}{c}-0.0113872 \\
(\mathrm{p}=0.8067)\end{array}$ \\
\hline USPIEM & $\begin{array}{l}-0.00476859 \\
(\mathrm{p}=0.6926)\end{array}$ & $\begin{array}{c}-0.0218701 \\
(\mathrm{p}=0.3739)\end{array}$ & $\begin{array}{c}-0.0778564 \\
(\mathrm{p}=0.5746)\end{array}$ & $\begin{array}{c}0.0317381 \\
(\mathrm{p}=0.1118)\end{array}$ & $\begin{array}{c}0.0269695 \\
(\mathrm{p}=0.2302)\end{array}$ \\
\hline USPIES & $\begin{array}{c}-0.0401248 \\
(\mathrm{p}=0.2349)\end{array}$ & $\begin{array}{c}0.0156031 \\
(\mathrm{p}=0.8201)\end{array}$ & $\begin{array}{c}-0.412495 \\
(\mathrm{p}=0.2854)\end{array}$ & $\begin{array}{c}0.0823290 \\
(\mathrm{p}=0.1396)\end{array}$ & $\begin{array}{c}0.0422042 \\
(\mathrm{p}=0.5008)\end{array}$ \\
\hline USPIED & $\begin{array}{c}0.0107748 \\
(\mathrm{p}=0.6820)\end{array}$ & $\begin{array}{l}-0.00877831 \\
(\mathrm{p}=0.8698)\end{array}$ & $\begin{array}{c}-0.253810 \\
(\mathrm{p}=0.4256)\end{array}$ & $\begin{array}{l}-0.00118354 \\
(\mathrm{p}=0.9782)\end{array}$ & $\begin{array}{c}0.00959123 \\
(\mathrm{p}=0.8444)\end{array}$ \\
\hline USPIERM & $\begin{array}{c}-0.000709017 \\
(\mathrm{p}=0.9471)\end{array}$ & $\begin{array}{c}0.0251140 \\
(p=0.2496)\end{array}$ & $\begin{array}{c}-0.0905536 \\
(p=0.4277)\end{array}$ & $\begin{array}{c}0.00491008 \\
(\mathrm{p}=0.7803)\end{array}$ & $\begin{array}{c}0.00420107 \\
(\mathrm{p}=0.8325)\end{array}$ \\
\hline USPIERS & $\begin{array}{c}0.0243413 \\
(\mathrm{p}=0.3455)\end{array}$ & $\begin{array}{c}-0.0113162 \\
(\mathrm{p}=0.8292)\end{array}$ & $\begin{array}{c}0.292308 \\
(\mathrm{p}=0.3275)\end{array}$ & $\begin{array}{c}0.000973074 \\
(\mathrm{p}=0.9817)\end{array}$ & $\begin{array}{c}0.0253144 \\
(\mathrm{p}=0.5973)\end{array}$ \\
\hline USPIERD & $\begin{array}{c}-0.0225716 \\
(\mathrm{p}=0.3965)\end{array}$ & $\begin{array}{c}-0.0752176 \\
(\mathrm{p}=0.1662)\end{array}$ & $\begin{array}{c}0.196933 \\
(\mathrm{p}=0.5324)\end{array}$ & $\begin{array}{c}0.0860470 \\
(\mathrm{p}=0.0510)\end{array}$ & $\begin{array}{c}0.0634754 \\
(\mathrm{p}=0.2004)\end{array}$ \\
\hline \multicolumn{6}{|c|}{ Share of innovative enterprises in total enterprises } \\
\hline No & 11 & 12 & 13 & 14 & 15 \\
\hline Constans & $\begin{array}{c}2.53385 \\
(\mathrm{p}=0.000)\end{array}$ & $\begin{array}{c}2.92964 \\
(\mathrm{p}=0.000)\end{array}$ & $\begin{array}{c}2.34128 \\
(\mathrm{p}=0.1703)\end{array}$ & $\begin{array}{c}5.27544 \\
(\mathrm{p}=0.000)\end{array}$ & $\begin{array}{c}3.20413 \\
(\mathrm{p}=0.000)\end{array}$ \\
\hline PIU & $\begin{array}{c}0.422498 \\
(\mathrm{p}=0.0225) \\
\end{array}$ & $\begin{array}{c}0.0662192 \\
(\mathrm{p}=0.8634)\end{array}$ & $\begin{array}{c}2.54998 \\
(\mathrm{p}=0.2154)\end{array}$ & $\begin{array}{c}0.0795699 \\
(\mathrm{p}=0.8019)\end{array}$ & $\begin{array}{c}0.502068 \\
(\mathrm{p}=0.1286)\end{array}$ \\
\hline PIUNP & $\begin{array}{c}-0.0150560 \\
(\mathrm{p}=0.7038)\end{array}$ & $\begin{array}{c}-0.0344396 \\
(\mathrm{p}=0.6782)\end{array}$ & $\begin{array}{c}0.651500 \\
(\mathrm{p}=0.1234)\end{array}$ & $\begin{array}{c}-0.199545 \\
(\mathrm{p}=0.0039)\end{array}$ & $\begin{array}{c}-0.214601 \\
(\mathrm{p}=0.0029)\end{array}$ \\
\hline PIUN & $\begin{array}{c}0.0184050 \\
(\mathrm{p}=0.3270)\end{array}$ & $\begin{array}{c}-0.0388911 \\
(p=0.3231)\end{array}$ & $\begin{array}{c}-0.243405 \\
(p=0.2193)\end{array}$ & $\begin{array}{c}0.101272 \\
(p=0.0020)\end{array}$ & $\begin{array}{c}0.119677 \\
(p=0.0005)\end{array}$ \\
\hline PIUNPROC & \begin{tabular}{|c|}
0.0511311 \\
$(\mathrm{p}=0.4103)$ \\
\end{tabular} & $\begin{array}{c}0.0344008 \\
(\mathrm{p}=0.7913)\end{array}$ & $\begin{array}{c}1.87674 \\
(\mathrm{p}=0.006)\end{array}$ & $\begin{array}{c}-0.100290 \\
(\mathrm{p}=0.3496)\end{array}$ & $\begin{array}{c}-0.0491585 \\
(\mathrm{p}=0.6585)\end{array}$ \\
\hline PIP & $\begin{array}{c}0.704103 \\
(\mathrm{p}=0.0210)\end{array}$ & $\begin{array}{c}-0.385094 \\
(\mathrm{p}=0.5438)\end{array}$ & $\begin{array}{c}7.27195 \\
(p=0.0389)\end{array}$ & $\begin{array}{c}-0.687136 \\
(\mathrm{p}=0.1894)\end{array}$ & $\begin{array}{c}0.0169664 \\
(\mathrm{p}=0.9750)\end{array}$ \\
\hline PIPNP & $\begin{array}{c}0.118153 \\
(\mathrm{p}=0.2573)\end{array}$ & $\begin{array}{c}0.227690 \\
(p=0.2975)\end{array}$ & $\begin{array}{c}-0.862668 \\
(p=0.4570)\end{array}$ & $\begin{array}{c}-0.299033 \\
(p=0.0975)\end{array}$ & $\begin{array}{c}-0.180880 \\
(p=0.3330)\end{array}$ \\
\hline PIPNPR & \begin{tabular}{|c|}
-0.0497395 \\
$(\mathrm{p}=0.3311)$ \\
\end{tabular} & $\begin{array}{l}-0.00246716 \\
(p=0.9816)\end{array}$ & $\begin{array}{c}0.220829 \\
(\mathrm{p}=0.6910)\end{array}$ & $\begin{array}{l}-0.00508137 \\
(\mathrm{p}=0.9541)\end{array}$ & $\begin{array}{c}-0.0548209 \\
(\mathrm{p}=0.5497)\end{array}$ \\
\hline PIPNPROC & $\begin{array}{c}-0.174195 \\
(\mathrm{p}=0.1267)\end{array}$ & $\begin{array}{c}-0.0608851 \\
(\mathrm{p}=0.7983)\end{array}$ & $\begin{array}{c}-0.772321 \\
(p=0.5443)\end{array}$ & $\begin{array}{c}0.243515 \\
(\mathrm{p}=0.2156)\end{array}$ & $\begin{array}{c}0.0693201 \\
(\mathrm{p}=0.7337)\end{array}$ \\
\hline UI & $\begin{array}{c}-1.20860 \\
(\mathrm{p}=0.0073)\end{array}$ & $\begin{array}{c}-0.130014 \\
(\mathrm{p}=0.8892)\end{array}$ & $\begin{array}{c}-11.2603 \\
(\mathrm{p}=0.0262)\end{array}$ & $\begin{array}{c}0,628733 \\
(\mathrm{p}=0,4138)\end{array}$ & $\begin{array}{c}-0.579871 \\
(\mathrm{p}=0.4678)\end{array}$ \\
\hline \multicolumn{6}{|c|}{ Input measures of innovation } \\
\hline No & 16 & 17 & 18 & 19 & 20 \\
\hline Constans & $\begin{array}{c}2.89554 \\
(\mathrm{p}=0.000)\end{array}$ & $\begin{array}{c}3.01865 \\
(\mathrm{p}=0.000)\end{array}$ & $\begin{array}{c}2.52858 \\
(\mathrm{p}=0.3518)\end{array}$ & $\begin{array}{c}1.09848 \\
(\mathrm{p}=0.0005)\end{array}$ & $\begin{array}{c}-0.611154 \\
(\mathrm{p}=0.1283)\end{array}$ \\
\hline NBR & $\begin{array}{c}0.272767 \\
(\mathrm{p}=0.0002)\end{array}$ & $\begin{array}{c}0.233844 \\
(\mathrm{p}=0.1352)\end{array}$ & $\begin{array}{c}1.54351 \\
(\mathrm{p}=0.0329)\end{array}$ & $\begin{array}{c}-0.515057 \\
(\mathrm{p}=0.000)\end{array}$ & $\begin{array}{c}-0.242290 \\
(\mathrm{p}=0.0257)\end{array}$ \\
\hline NPRAC & $\begin{array}{c}-0.0310933 \\
(\mathrm{p}=0.4030)\end{array}$ & $\begin{array}{c}-0.0748157 \\
(\mathrm{p}=0.3642)\end{array}$ & $\begin{array}{l}-0.00497047 \\
(\mathrm{p}=0.9895)\end{array}$ & $\begin{array}{c}0.526484 \\
(\mathrm{p}=0.000)\end{array}$ & $\begin{array}{c}0.495390 \\
(\mathrm{p}=0.000)\end{array}$ \\
\hline
\end{tabular}


Innovation and Entrepreneurship in a Space-time...

\begin{tabular}{|l|c|c|c|c|c|}
\hline NWN & $\begin{array}{c}0.0423248 \\
(\mathrm{p}=0.0272)\end{array}$ & $\begin{array}{c}0.0455428 \\
(\mathrm{p}=0.2804)\end{array}$ & $\begin{array}{c}0.0550374 \\
(\mathrm{p}=0.7817)\end{array}$ & $\begin{array}{c}0.00986768 \\
(\mathrm{p}=0.6620)\end{array}$ & $\begin{array}{c}0.0521925 \\
(\mathrm{p}=0.0742)\end{array}$ \\
\hline NŚT & $\begin{array}{c}-0.326841 \\
(\mathrm{p}=0.000)\end{array}$ & $\begin{array}{c}-0.313573 \\
(\mathrm{p}=0.0432)\end{array}$ & $\begin{array}{c}-1.41582 \\
(\mathrm{p}=0.0514)\end{array}$ & $\begin{array}{c}-0.0252684 \\
(\mathrm{p}=0.7596)\end{array}$ & $\begin{array}{c}-0.352109 \\
(\mathrm{p}=0.0012)\end{array}$ \\
\hline
\end{tabular}

Source: own development

As indicated by the data in Table 2, the parameters of only one primary function were statistically significant. In the case of the others, the significance factor exceeded the adopted minimum value. For this reason, the values of the regression function parameters were overestimated. As a result, further statistically insignificant independent variables were removed. Starting the removal process from the variables with the highest value of the $\mathrm{p}$-factor, independent variables were removed one by one until all other variables were statistically significant. As a consequence, the parameters of the function, presented in Table 3, were obtained.

Table 3. Parameters of the final regression functions ${ }^{*}$

\begin{tabular}{|c|c|c|c|c|c|}
\hline $\begin{array}{c}\text { Independent } \\
\text { variables }\end{array}$ & ST & SU & SN & NPRZ & NSU \\
\hline \multicolumn{6}{|c|}{ Net revenues from sales of innovations } \\
\hline Lp. & 1 & 2 & 3 & 4 & 5 \\
\hline Constans & $\begin{array}{c}2.12591 \\
(\mathrm{p}=0.000)\end{array}$ & $\begin{array}{c}2.22932 \\
(\mathrm{p}=0.000)\end{array}$ & $\mathrm{X}$ & $\begin{array}{c}4.13182 \\
(\mathrm{p}=0.000)\end{array}$ & $\begin{array}{c}1.66761 \\
(p=0.000)\end{array}$ \\
\hline USPI & $\begin{array}{c}0.180763 \\
(\mathrm{p}=0.0093)\end{array}$ & $\mathrm{X}$ & $\mathrm{X}$ & $\begin{array}{c}0.335799 \\
(p=0.0021)\end{array}$ & $\begin{array}{c}0.521765 \\
(p=0.000)\end{array}$ \\
\hline USPIR & $\begin{array}{c}-0.102409 \\
(\mathrm{p}=0.0229)\end{array}$ & $\mathrm{X}$ & $\mathrm{X}$ & $\begin{array}{c}-0.295882 \\
(p=0.0002)\end{array}$ & $\begin{array}{l}-0.412638 \\
(p=0.000)\end{array}$ \\
\hline USPIP & $\begin{array}{c}-0.0810192 \\
(\mathrm{p}=0.0238)\end{array}$ & $\mathrm{X}$ & $\mathrm{X}$ & $\begin{array}{c}-0.149964 \\
(p=0.0082)\end{array}$ & $\begin{array}{c}-0.234686 \\
(p=0.0003)\end{array}$ \\
\hline USPIE & $\mathrm{X}$ & $\mathrm{X}$ & $\mathrm{X}$ & $\begin{array}{c}0.110806 \\
(p=0.0003)\end{array}$ & $\mathbf{X}$ \\
\hline USPIER & $\mathrm{X}$ & $\mathrm{X}$ & $\mathrm{X}$ & $\mathbf{X}$ & $\begin{array}{c}0.123458 \\
(p=0.0005)\end{array}$ \\
\hline USPT & $\begin{array}{c}-0.0274025 \\
(\mathrm{p}=0.0821)\end{array}$ & $\begin{array}{c}-0.0732865 \\
(\mathrm{p}=0.0087)\end{array}$ & $\mathrm{X}$ & $\begin{array}{c}0.0871210 \\
(\mathbf{p}=0.0007)\end{array}$ & $\begin{array}{c}0.0572240 \\
(p=0.0488)\end{array}$ \\
\hline $\begin{array}{l}\text { Skorygowany } \\
\text { R2 }\end{array}$ & 0.0511 & 0.0307 & $\mathrm{X}$ & 0.1535 & 0.1334 \\
\hline \multicolumn{6}{|c|}{ Net revenues from sales of innovations by size classes } \\
\hline Lp. & 6 & 7 & 8 & 9 & 10 \\
\hline Constans & $\begin{array}{c}2.03128 \\
(\mathrm{p}=0.000)\end{array}$ & $\begin{array}{c}1.90728 \\
(\mathrm{p}=0.000)\end{array}$ & $\begin{array}{c}0.179019 \\
(\mathrm{p}=0.4661)\end{array}$ & $\begin{array}{c}4.70842 \\
(p=0.000)\end{array}$ & $\begin{array}{c}2.30596 \\
(p=0.000)\end{array}$ \\
\hline USPIM & $\mathrm{X}$ & $\mathrm{X}$ & $\begin{array}{l}-0.307495 \\
(\mathrm{p}=0.037)\end{array}$ & $\begin{array}{l}-0.0700129 \\
(p=0.0017)\end{array}$ & $\begin{array}{l}-0.0713702 \\
(p=0.0067)\end{array}$ \\
\hline USPIS & $\mathrm{X}$ & $\mathrm{X}$ & $\mathrm{X}$ & $\mathbf{X}$ & $\begin{array}{c}-0.146031 \\
(p=0.0052)\end{array}$ \\
\hline USPID & $\begin{array}{c}0.147890 \\
(\mathrm{p}=0.0021)\end{array}$ & $\begin{array}{c}0.0773456 \\
(\mathrm{p}=0.0239)\end{array}$ & $\mathrm{X}$ & $\mathbf{X}$ & $\begin{array}{c}0.0795524 \\
(p=0.0015)\end{array}$ \\
\hline
\end{tabular}




\begin{tabular}{|c|c|c|c|c|c|}
\hline USPIRM & $\mathrm{X}$ & $\begin{array}{c}-0.0489797 \\
(\mathrm{p}=0.0344)\end{array}$ & $\begin{array}{c}0.291897 \\
(\mathrm{p}=0.0053)\end{array}$ & $\mathbf{X}$ & $\mathbf{X}$ \\
\hline \begin{tabular}{|l|} 
USPIRS \\
\end{tabular} & $\mathrm{X}$ & $\mathrm{X}$ & $\mathrm{X}$ & $\mathbf{X}$ & $\mathbf{X}$ \\
\hline USPIRD & $\begin{array}{c}-0.0824047 \\
(\mathrm{p}=0.0059)\end{array}$ & $\mathrm{X}$ & $\mathrm{X}$ & $\mathbf{X}$ & $\mathbf{X}$ \\
\hline USPIPM & $\mathrm{X}$ & $\mathrm{X}$ & $\mathrm{X}$ & $\mathbf{X}$ & $\mathbf{X}$ \\
\hline USPIPS & $\begin{array}{c}0.0351849 \\
(\mathrm{p}=0.0784)\end{array}$ & $\mathrm{X}$ & $\begin{array}{c}0.428746 \\
(\mathrm{p}=0.0179)\end{array}$ & $\begin{array}{l}-0.142440 \\
(p=0.000)\end{array}$ & $\mathbf{X}$ \\
\hline USPIPD & $\begin{array}{c}-0.0621435 \\
(\mathrm{p}=0.0096)\end{array}$ & $\mathrm{X}$ & $\mathrm{X}$ & $\begin{array}{c}0.0374552 \\
(p=0.0153)\end{array}$ & $\mathbf{X}$ \\
\hline USPIEM & $\mathrm{X}$ & $\begin{array}{c}-0.0371041 \\
(\mathrm{p}=0.0354)\end{array}$ & $\mathrm{X}$ & $\begin{array}{c}0.0453301 \\
(p=0.0004)\end{array}$ & $\begin{array}{c}0.0489678 \\
(p=0.0010)\end{array}$ \\
\hline USPIES & $\begin{array}{c}-0.0372231 \\
(\mathrm{p}=0.0249) \\
\end{array}$ & $\mathrm{X}$ & $\mathrm{X}$ & $\begin{array}{c}0.0675872 \\
(p=0.0091) \\
\end{array}$ & $\begin{array}{c}0.0682403 \\
(p=0.0569)\end{array}$ \\
\hline \begin{tabular}{|l|} 
USPIED \\
\end{tabular} & $\mathrm{X}$ & $\mathrm{X}$ & $\mathrm{X}$ & $\mathbf{X}$ & $\mathbf{X}$ \\
\hline USPIERM & $\mathrm{X}$ & $\begin{array}{c}0.0371447 \\
(\mathrm{p}=0.0418)\end{array}$ & $\mathrm{X}$ & $\mathbf{X}$ & $\mathbf{X}$ \\
\hline \begin{tabular}{|l} 
USPIERS \\
\end{tabular} & $\mathrm{X}$ & $\mathrm{X}$ & $\mathrm{X}$ & $\mathrm{X}$ & $\mathbf{X}$ \\
\hline USPIERD & $\mathrm{X}$ & $\begin{array}{c}-0.0873478 \\
(\mathrm{p}=0.0011)\end{array}$ & $\mathrm{X}$ & $\begin{array}{c}0.0553177 \\
(\mathbf{p}=\mathbf{0 . 0 0 0 9 )}\end{array}$ & $\mathbf{X}$ \\
\hline \begin{tabular}{|l|} 
R2 \\
\end{tabular} & 0.048 & 0.0894 & 0.0732 & 0.2487 & 0.1411 \\
\hline \multicolumn{6}{|c|}{ Share of innovative enterprises in total enterprises } \\
\hline Lp. & 11 & 12 & 13 & 14 & 15 \\
\hline Constans & $\begin{array}{c}2.50962 \\
(p=0.000)\end{array}$ & $\begin{array}{c}2.71292 \\
(\mathrm{p}=0.000)\end{array}$ & $\begin{array}{c}2.66472 \\
(p=0.0281)\end{array}$ & $\begin{array}{c}5.04718 \\
(p=0.000)\end{array}$ & $\begin{array}{c}3.20071 \\
(p=0.000)\end{array}$ \\
\hline PIU & $\begin{array}{c}0.417103 \\
(p=0.0002)\end{array}$ & $\mathrm{X}$ & $\mathbf{X}$ & $\mathbf{X}$ & $\begin{array}{c}0.368412 \\
(p=0.0017)\end{array}$ \\
\hline PIUNP & $\mathbf{X}$ & $\mathrm{X}$ & $\mathbf{X}$ & $\begin{array}{c}-0.139158 \\
(p=0.0177)\end{array}$ & $\begin{array}{c}-0.204011 \\
(p=0.0017)\end{array}$ \\
\hline PIUNPR & $\mathbf{X}$ & $\mathrm{X}$ & $\mathbf{X}$ & $\begin{array}{c}0.106764 \\
(p=0.0005) \\
\end{array}$ & $\begin{array}{c}0.114983 \\
(p=0.0004)\end{array}$ \\
\hline PIUNPROC & $\mathbf{X}$ & $\mathrm{X}$ & $\begin{array}{c}1.36057 \\
(p=0.000)\end{array}$ & $\mathbf{X}$ & $\mathbf{X}$ \\
\hline PIP & $\begin{array}{c}0.69259 \\
(p=0.0018)\end{array}$ & $\begin{array}{c}-0.244344 \\
(\mathrm{p}=0.000)\end{array}$ & $\mathbf{X}$ & $\mathbf{X}$ & $\mathbf{X}$ \\
\hline PIPNP & $\mathbf{X}$ & $\mathrm{X}$ & $\mathbf{X}$ & $\begin{array}{c}-0.495988 \\
(p=0.000)\end{array}$ & $\begin{array}{c}-0.259125 \\
(p=0.0521)\end{array}$ \\
\hline PIPNPR & $\mathbf{X}$ & $\mathrm{X}$ & $\mathbf{X}$ & $\mathbf{X}$ & $\mathbf{X}$ \\
\hline PIPNPROC & $\begin{array}{c}-0.195188 \\
(p=0.0595)\end{array}$ & $\mathrm{X}$ & $\mathbf{X}$ & $\mathbf{X}$ & $\mathbf{X}$ \\
\hline UI & $\begin{array}{c}-1.04845 \\
(p=0.0008)\end{array}$ & $\mathrm{X}$ & $\begin{array}{c}-1.93326 \\
(p=0.0017)\end{array}$ & $\begin{array}{c}0.341561 \\
(p=0.0046)\end{array}$ & $\begin{array}{c}-0.382138 \\
(p=0.0894)\end{array}$ \\
\hline R2 & 0.1926 & 0.0902 & 0.1143 & 0.17 & 0.2647 \\
\hline \multicolumn{6}{|c|}{ Input measures of innovation } \\
\hline Lp. & 16 & 17 & 18 & 19 & 20 \\
\hline Constans & $\begin{array}{c}2.69677 \\
(p=0.000)\end{array}$ & $\begin{array}{c}2.19708 \\
(\mathrm{p}=0.000)\end{array}$ & $\begin{array}{c}2.36858 \\
(\mathrm{p}=0.0281)\end{array}$ & $\begin{array}{c}1.00616 \\
(p=0.000)\end{array}$ & $\begin{array}{c}-0.611154 \\
(p=0.1283)\end{array}$ \\
\hline NBR & $\begin{array}{c}0.253121 \\
(p=0.0002)\end{array}$ & $\mathrm{X}$ & $\begin{array}{c}1.54963 \\
(\mathrm{p}=0.0211)\end{array}$ & $\begin{array}{c}-0.531757 \\
(p=0.000)\end{array}$ & $\begin{array}{c}-0.242290 \\
(p=0.0257)\end{array}$ \\
\hline
\end{tabular}




\begin{tabular}{|l|c|c|c|c|c|}
\hline NPRAC & $\mathbf{X}$ & $\mathrm{X}$ & $\mathrm{X}$ & $\begin{array}{c}\mathbf{0 . 5 3 4 9 3 5} \\
(\mathbf{p}=\mathbf{0 . 0 0 0})\end{array}$ & $\begin{array}{c}\mathbf{0 . 4 9 5 3 9 0} \\
(\mathbf{p}=\mathbf{0 . 0 0 0})\end{array}$ \\
\hline NWN & $\begin{array}{c}\mathbf{0 . 0 3 4 0 3 4 1} \\
(\mathbf{p}=\mathbf{0 . 0 3 7 0})\end{array}$ & $\mathrm{X}$ & $\mathrm{X}$ & $\mathbf{X}$ & $\begin{array}{c}\mathbf{0 . 0 5 2 1 9 2 5} \\
(\mathbf{p}=\mathbf{0 . 0 7 4 2})\end{array}$ \\
\hline NŚT & $\begin{array}{c}-\mathbf{0 . 3 2 8 6 7 6} \\
(\mathbf{p}=\mathbf{0 . 0 0 0})\end{array}$ & $\begin{array}{c}-0.0945593 \\
(\mathrm{p}=0.0066)\end{array}$ & $\begin{array}{c}-1.32351 \\
(\mathrm{p}=0.0455)\end{array}$ & $\mathbf{X}$ & $\begin{array}{c}-\mathbf{0 . 3 5 2 1 0 9} \\
(\mathbf{p}=\mathbf{0 . 0 0 1 2})\end{array}$ \\
\hline R2 & $\mathbf{0 . 1 2 4 3}$ & 0.0444 & 0.0368 & $\mathbf{0 . 6 3 6 7}$ & $\mathbf{0 . 5 0 2 5}$ \\
\hline
\end{tabular}

$\mathrm{X}$ - variable withdrawn from the regression function equation, ${ }^{*}$ - functions accepted for further analysis are given in bold

Source: own development

Of the 24 measures of innovation initially adopted, five were found to be statistically insignificant in relation to none of the regression functions (USPIRS, USPIPM, USPIED, USPIERD, PIPNPR), and the next seven variables were only relevant in explaining one function (USPIE, USPIER, ENTRY, USPIRD, USPIERM, PIUNPROC, PIPNPROC).

Data contained in Table 3 indicate that, in general, R2 coefficients are not too high; the highest value for one function is (0.6367). At the same time, a total of 9 regression functions were withdrawn from further inference due to the insufficient level of explanation of the studied phenomenon (removed functions are 1, 2, 3, 6, 7, 8, 12, 17, 18). As the results indicate, the rate of failure of enterprises is not explained by the measures of innovation in any of the adopted cases.

The two measures of entrepreneurship that respond most strongly to innovation activity, both from the input and output sides, include saturation with enterprises and saturation with start-ups. This can be concluded based on the number of statistically significant regression functions as well as on a comparison of function determination coefficients. Out of 11 regression functions finally accepted for inference, 8 explained the variability of saturation measures: 4 saturation with enterprises (functions $4,9,14$, 19 ) and 4 with newly established companies (functions $5,10,15,20$ ). At the same time, from the four dimensions of innovation adopted, each proved to explain these measures of entrepreneurship.

The other two measures of entrepreneurship are less explained by innovation activity. The rate of creation of start-ups can be explained by two approaches to innovative activity, i.e. by an input measure and one of the output measures (functions 11, 16). In contrast, the net enterprise rate is explained only by one regression function estimated using one of the outcome measures (function 13).

The obtained results were analysed from the perspective of innovation measures. It turned out that the broadest spectrum of impact on entrepreneurship is the output innovation seen as the share of innovative enterprises in the total number of business entities. This measure was statistically significant in explaining the largest number of dependent variables, as it explained 4 out of 5 adopted entrepreneurship indicators, i.e. saturation with enterprises, saturation with newly established companies, the rate of creation of new companies and the net rate (functions 11, 13, 14, 15).

However, looking at the values of the coefficients of determination, the input expenditure measure of innovation best explains the phenomena related to entrepreneurship (especially functions 19,20 ). 
The next stage of the analysis is to compare the values of the parameters of the regression function from the point of view of their relative and absolute value to determine the direction and strength of interactions. Considering the first group of regression functions, in which the output measures of innovation in the form of the share of revenues from sales of innovations (functions 4, 5) were independent variables, the largest absolute values were at the parameters presenting the share of net sales from innovations in total revenues (USPI) and the share of net sales from market innovations in total revenues (USPIR). The most substantial impact of these measures was on both saturation with enterprises and saturation with start-ups. At the same time, judging by the relative value, USPI has a directly proportional effect, and USPIR inversely proportional to both dependent variables.

It can, therefore, be assumed that, when looking at the sales of innovative products in total, the higher the share of revenues from their sales, the higher the saturation of a given region with enterprises in total and in new companies. As a general conglomerate, the sale of innovative products in total attracts new companies to the region and allows for the maintenance of existing enterprises. Thus it can be a factor attracting entrepreneurship. However, at a higher level of detail, it turns out that the greater the share of sales of innovative products for markets, the lower the saturation with total and newly created enterprises. It can, therefore, be assumed that if innovations are new at the level of the entire market, then their sale may constitute a barrier to entry for new companies or exert competitive pressure for existing companies.

When looking at the share of sales of innovative products dependant on the size of enterprises entering this market, further relationships can be observed (functions 9, 10). The share of sales of innovative products in small, medium and large enterprises only affected saturation with existing and new companies. When analysing the absolute values of the parameters with these regression functions, it can be seen that they are significantly lower than in the case of the previously analysed functions 4 and 5. Innovation measures related to medium-sized enterprises show the highest parameter values. Saturation with enterprises is most strongly affected by innovation measured by the share of sales of innovative products for the enterprise by medium-sized companies (USPIPS), while saturation with start-ups is most strongly influenced by the share of sales of innovative products in medium-sized enterprises (USPIS). The impact of both variables is negative, which may indicate the innovative activity of medium-sized enterprises, and this is a barrier to new companies entering the market while creating competitive pressure for stable companies. However, the low absolute value of the parameters with the regression function indicates that the independent variables are not very strongly affected by dependent variables.

Thirdly, the impact of the number of innovative enterprises, and the share of innovative enterprises on the market was examined. This aspect turned out to be the most important from the number of regression functions accepted for inference; four were accepted (functions 11, 13,14, 15). The most important variable explaining the measures of entrepreneurship is the share of innovative enterprises in the total number of enterprises (UI) because the parameters of this variable had the highest absolute value, especially concerning the rates of creation of new enterprises and net rates. This parameter has negative values, which means that the higher the share of innovative enterprises, the lower the creation and net enterprise rates. It can, therefore, be assumed that the existence of innovative enterprises is a barrier to entry for new companies. Besides, the rate of creation of 
new enterprises is relatively strongly and positively influenced by the share of innovative enterprises in the industry (PIP) and in the services sector (PIU). On the other hand, the net rate is strongly influenced by the share of service enterprises introducing new or significantly improved processes (PIUNPROC).

The impact of this aspect of innovation on saturation with enterprises and start-ups is lower than in relation to the rates. Also, in the case of these measures, the share of innovative enterprises in the total number (UI) turned out to be significant. Besides, saturation with enterprises is explained by the participation of industrial enterprises introducing new or significantly improved products (PIPNP), and saturation with new enterprises by the participation of innovative enterprises in the services sector (PIU).

The last aspect of innovation analysed is the input aspect related to expenditure on innovation. From the perspective of the R 2 coefficient of determination, this aspect seems to have the strongest impact on entrepreneurial activity, especially concerning saturation with enterprises and newly created companies. Among entrepreneurship rates, this aspect of innovation only affects the rate at which new businesses are created.

Expenditures on innovation activities in relation to gross fixed capital formation (NST) is the variable most strongly affecting the rate of creation of new enterprises. Saturation with enterprises is comparable to the share of expenditure on $R \& D$ activity in GDP (NBR) and expenditure on R\&D activity per professionally active person (NPRAC). NPRAC is also the factor with the strongest and most positive impact on saturation with start-ups.

Expenditure on innovation activities in national expenditure (NWN) may affect the rate of creation and saturation with new companies, but this impact is so low that it can be ignored in further considerations.

\section{Final remarks}

The study of the relationship between innovation and entrepreneurship encounters the problem of measuring both phenomena. Both are broad concepts; hence depending on the degree of detail of their measures, different, ambiguous pictures can be obtained. The article has aimed to determine the impact of innovation on the entrepreneurial process depending on the measures adopted. At the initial stage as many innovation and entrepreneurship measures as possible were adopted, in order to eliminate statistically insignificant relationships in subsequent research stages and obtain a complete picture. The survey covered Polish voivodeships on an annual basis in the years 2003-2018.

The results obtained allow for some observations. First of all, it has been confirmed that due to the breadth of understanding and the measurement of innovation and entrepreneurship, the results of the dependency studies depend on the measures adopted and different results can be obtained. Besides, saturation with enterprises and saturation with newly created companies seem to be more sensitive to innovation than other measures of entrepreneurship. At the same time in terms of input, measured by expenditure on R\&D, there is a stronger impact on entrepreneurship than innovations in the output system, related to revenues from innovative activities, i.e. the scale of enterprises implementing innovations. Generally, however, the impact of innovation on entrepreneurship, regardless of its measures, is not strong and does not affect certain aspects at all. It confirms the belief among researchers of the complexity of both phenomena. 


\section{References}

Acs, Z.J., Audretsch, D.B. (1988). Innovation in Large and Small Firms: An Empirical Analysis. The American Economic Review, 78, 678-690.

Armington, C., Acs, Z.J. (2002). The Determinants of Regional Variation in New Firm Formation. Regional Studies, 36, 33-45. doi: 10.1080/00343400120099843

Arvanitis, S., Stucki, T. (2012). What determines the innovation capability of firm founders?. Industrial and Corporate Change, 21(4), 1049-1084. doi: 10.1093/icc/dts003

Audretsch, D.B., Fritsch, M. (2002). Growth Regimes over Time and Space. Regional Studies, 36(2), 113-124.

Auerswald, P.E. (2008). Entrepreneurship in the Theory of the Firms. Small Business Economics, 30, $111-126$.

Bhattacharya, M., Bloch, H. (2004). Determinants of Innovation. Small Business Economics, 22, 155-162.

Box, M. (2008). The death of firms: exploring the effects of environment and birth cohort on firm survival in Sweden. Small Business Economics, 31, 379-393.

Caliendo, M., Fosse, F.M., Kritikos, A.S. (2009). Risk attitudes of nascent entrepreneurs- new evidence from an experimentally validated survey. Small Business Economics, 32, 153-167.

Capelleras, J.-L., Greene, F.J. (2008). The determinants and growth implications of venture creation speed. Entrepreneurship and Regional Development, 20, 317-343.

de Silva, D.G., McComb, R. (2012). Research Universities and Regional High-Tech Firm Start-Up and Exit. Economic Inquiry, 50(1), 112-130. doi: 10.1111/j.1465-7295.2010.00353.x

Esteve-Pérez, S., Mañez-Castillejo, J.A. (2008). The Resource-Based Theory of the Firm and Firm Survival. Small Business Economics, 30, 231-249.

Fritsch, M., Schroeter, A. (2011). Why does the effect of new business formation differ across regions? Small Business Economics, 36, 383-400. doi: 10.1007/s11187-009-9256-9

Gaweł, A. (2013). Proces przedsiębiorczy. Tworzenie nowych przedsiebiorstw. Warszawa: Wydawnictwo Difin.

Honjo, Y., Kato, M., Okamuro, H. (2014). R\&D investment of start-up firms: does founders' human capital matter?. Small Business Economics, 42, 207-220. doi: 10.1007/s11187-013-9476-X

Huergo, E., Jaumandreu, J. (2004). How Does Probability of Innovation Change with Firm Age?. Small Business Economics, 22, 193-207.

Koellinger, P. (2008). Why are some entrepreneurs more innovative than others?. Small Business Economics, 31, 21-37.

Korunka, Ch., Frank, H., Lueger, M., Mugler, J. (2003). The Entrepreneurial Personality in the Context of Resources, Environment, and the Startup Process - A Configurational Approach. Entrepreneurship Theory and Practice, 28, 23-42.

Lejpras, A. (2014). How innovative are spin-offs at later stages of development? Comparing innovativeness of established research spin-offs and otherwise created firms. Small Business Economics, 43, 327-351. doi: 10.1007/s11187-013-9534-4

Lin, P.Ch., Huang, D.S. (2008). Technological Regimes and Firm Survival: Evidence Across Sectors and Over Time. Small Business Economics, 30, 175-186.

Luttmer, E.G.J. (2007). Selection, Growth, and the Size Distribution of Firms. The Quarterly Journal of Economics, 122(3), 1103-1144.

Mengistae, T. (2006). Competition and Entrepreneurs' Capital in Small Business Longevity and Growth. Journal of Development Studies, 42, 812-836.

Naudé, W., Gries, T., Wood E., Meintjies A. (2008). Regional determinants of entrepreneurial start-up in a developing country. Entrepreneurship and Regional Development, 20, 111-124. 
Nauwelaers, C., Wintjes, R. (2002). Innovating SMEs and Regions: The Need for Policy Intelligence and Interactive Policies. Technology Analysis and Strategic Management, 14(2), 201-215.

North, D., Smallbone, D. (2000). Innovative Activity in SMEs and Rural Economic Development: Some Evidence from England. European Planning Studies, 8(1), 87-106.

Paulson, A.L., Townsend, P.M., Karaivanov, A. (2006). Distinguishing Limited Liability from Moral Hazard in a Model of Entrepreneurship. Journal of Political Economy, 114, 100-144.

Peneder, M.R. (2008). Firm entry and turnover: the nexus with profitability and growth. Small Business Economics, 30, 327-344.

Schäfer, D., Talavera, O. (2009). Small business survival and inheritance: evidence from Germany. Small Business Economics, 32, 95-109.

Schumpeter, J. (1960). Teoria rozwoju gospodarczego. Warszawa: PWN.

Simonen, J., McCann, P. (2008). Innovation, R\&D cooperation and labor recruitment: evidence from Finland. Small Business Economics, 31, 181-194.

Strotmann, H. (2007). Entrepreneurial Survival. Small Business Economics, 28, 87-104.

Tsvetkova, A., Thill, J.C, Strumsky, D. (2014). Metropolitan innovation, firm size, and business survival in a high-tech industry. Small Business Economics, 43, 661-676. doi: 10.1007/s11187-014-9550-z

Wagner, J., Sternberg, R. (2004). Start-up activities, industrial characteristics, and the regional milieu: Lessons for entrepreneurship support policies from German micro data. The Annals of Regional Science, 38, 219-240.

Wang, J. (2014). R\&D activities in start-up firms: What can we learn from founding resources?. Technology Analysis \& Strategic Management, 26(5), 517-529. doi: 10.1080/09537325.2013.870990

Yang, C., Bossink, B., Peverelli, P. (2017). High-tech start-up firm survival originating from a combined use of internal resources. Small Business Economics, 49, 799-824. doi: 10.1007/s11187-017-9858-6

Aleksandra Gawel, full professor, Poznan University of Economics and Business, Institute of International Business and Economics, Department of International CompetitivnessProfessor of economics working at Poznan University of Economics and Business; university teacher and researcher; lecturer in the areas of: Entrepreneurship, Business Simulation Games, Microeconomics; author or co-author of over 120 publications on: innovation, entrepreneurship, entrepreneurial education, the business cycle, the labour market, regional development; participant in several national and international projects; member of editorial board of "Studia Oeconomica Posnaniensia"; member of scientific committees at international conferences; reviewer of national and international journals; supervisor of $\mathrm{PhD}$ and master projects.

ORCID: 0000-0002-7426-3474

\section{Address:}

Uniwersytet Ekonomiczny w Poznaniu

Katedra Konkurencyjności Międzynarodowej

al. Niepodległości 10

61-875 Poznań, Poland

e-mail: aleksandra.gawel@ue.poznan.pl 